HWS-92/06

\title{
Quantum Mechanics with Explicit Time Dependence
}

\author{
John Rogers* and Donald Spector ${ }^{\dagger}$ \\ Department of Physics, Eaton Hall \\ Hobart and William Smith Colleges \\ Geneva, NY 14456 USA
}

\begin{abstract}
We investigate quantum mechanical Hamiltonians with explicit time dependence. We find a class of models in which an analogue of the time independent Schrödinger equation exists. Among the models in this class is a new exactly soluble model, the harmonic oscillator with frequency inversely proportional to time.
\end{abstract}

July 1992

* Address after Sept. 1, 1992: Department of Applied Physics, Columbia University, New York, NY 10027 USA

$\dagger$ spector@hws.bitnet 
In this paper, we study quantum mechanical systems in which the Hamiltonian has an explicit dependence on time. As is well-known, when a Hamiltonian is independent of time, the solution of the corresponding quantum system can be reduced, via separation of variables, to solving the time-independent Schrödinger equation (see for example [1]). When the Hamiltonian depends on time, such a simplification does not generically occur. In this paper, we will identify a class of time-dependent Hamiltonians for which an analogue of the time-independent Schrödinger equation does exist. We stress that in our work, the time dependence is treated exactly; we do not assume the time dependence to be adiabatic or perturbative. Among the models we consider, we find a new exactly soluble model: the harmonic oscillator with frequency inversely proportional to time.

Time-dependent Hamiltonians arise in a variety of situations, ranging from laboratory setups in which the experimenter dynamically modifies the environment, to situations in which the strength of an interaction depends on the expectation value of field which has not yet reached (or, in the case of a runaway potential, might never reach) equilibrium. In the context of cosmology, Dirac proposed that the Newtonian gravitational constant might depend on time, with the implication that gravity is weak because the universe is old [2]. Formally, the problem of determining the excitation spectrum of certain time-dependent solitons can be formulated as solving particular quantum systems with time-dependent Hamiltonians [3].

Our approach will be first to identify a class of time-dependent models in which one can obtain an analogue of the time-independent Schrödinger equation, and then to study one system in detail, the harmonic oscillator with frequency inversely proportional to time. For the sake of simplicity, we will restrict our attention in this paper to systems with only one spatial dimension, but it will be readily apparent that our techniques can be applied to higher dimensional models as well.

The Schrödinger equation in position space reads

$$
\mathbf{H} \Psi(x, t)=i \hbar \frac{\partial \Psi(x, t)}{\partial t} .
$$

Let us consider the simplest class of time-dependent Hamiltonians,

$$
\mathbf{H}=-\frac{\hbar^{2}}{2 m} \frac{\partial^{2}}{\partial x^{2}}+g(t) x^{n}
$$

As long as $g(t)$ is real, this Hamiltonian is hermitian. Of course, separation of variables in terms of $x$ and $t$ will not be fruitful in this case, so we attempt to find a change of 
variables for which separation of variables will be useful. To this end, we define a new variable $y=f(t) x$. The time-dependent Schrödinger equation now reads

$$
-\frac{\hbar^{2}}{2 m} f(t)^{2} \frac{\partial^{2}}{\partial y^{2}} \widetilde{\Psi}(y, t)+\frac{g(t)}{f(t)^{n}} y^{n} \widetilde{\Psi}(y, t)=i \hbar \frac{\dot{f}(t)}{f(t)} y \frac{\partial}{\partial y} \widetilde{\Psi}(y, t)+i \hbar \frac{\partial \widetilde{\Psi}(y, t)}{\partial t} .
$$

We use $\widetilde{\Psi}$ to denote the wavefunction as a function of $y$ and $t$. Obviously, $\Psi(x, t)=$ $\widetilde{\Psi}(y(x, t), t)$. Note that in (3), the partial derivatives with respect to time are now taken with $y$ fixed rather than with $x$ fixed.

Inspecting (3), it is easy to see that separation of variables in $y$ and $t$ will work provided that we choose

$$
f(t)=(p t+q)^{-1 / 2}
$$

and that

$$
g(t)=(p t+q)^{-(2+n) / 2}
$$

where $p$ and $q$ are constants. Hereon, we take (4) and (5) to hold. By considering solutions to (3) of the form $\widetilde{\Psi}(y)=T(t) Y(y)$, we see that the general solution to the Schrödinger equation can be written as

$$
\widetilde{\Psi}(y, t)=\sum_{k} c_{k} T_{k}(t) Y_{k}(y)
$$

where the $c_{k}$ are arbitrary constants, and where $Y_{k}$ and $T_{k}$ are the solutions of, respectively,

$$
-\frac{d^{2}}{d y^{2}} Y_{k}+i \frac{m p}{\hbar} y \frac{d}{d y} Y_{k}+\frac{2 m}{\hbar^{2}} y^{n} Y_{k}=\gamma_{k} Y_{k}
$$

and

$$
\frac{2 m i}{\hbar}(p t+q) \frac{d}{d t} T_{k}=\gamma_{k} T_{k}
$$

with $\gamma_{k}$ a constant.

The functions $Y_{k}$ are formally analogous to the stationary states of ordinary quantum mechanics, but are not states of definite energy. The constants $\gamma_{k}$ characterize these basic solutions but, again in contrast to the ordinary case, these constants do not correspond to the energies of the associated states. Indeed, the $\gamma_{k}$ are complex, with $\operatorname{Im}\left(\gamma_{k}\right)$ the same for all $k$.

The differential equation for $T_{k}$ can be solved exactly, yielding

$$
T_{k}(t)=\left(1+\frac{p t}{q}\right)^{-\left(i \hbar \gamma_{k} / 2 m p\right)}
$$


where we have chosen the normalization $T_{k}(0)=1$. Note that since the $\gamma_{k}$ are not real, the $T_{k}$ are not pure phase.

Thus, we have found a class of time-dependent Hamiltonians for which separation of variables is useful. For these systems, one recovers a mathematical structure formally analogous to the one one finds for time-independent systems. In particular, if we define the pseudo-Hamiltonian

$$
\widetilde{\mathcal{H}}=-\frac{d^{2}}{d y^{2}}+i \frac{m p}{\hbar} y \frac{d}{d y}+\frac{2 m}{\hbar^{2}} y^{n},
$$

we have reduced the problem of solving the time-dependent Schrödinger equation to finding the eigenfunctions and eigenvalues of $\widetilde{\mathcal{H}}$. The most significant difference between the case we consider and the ordinary case of time-independent potentials is this: in the ordinary case, the operator whose eigenfunctions and eigenvalues we must find has exactly the same form as the Hamiltonian; in our case, the operator whose eigenfunctions and eigenvalues we must find is not the Hamiltonian. (Note that in the limit that $p=0$ the pseudoHamiltonian becomes the ordinary Hamiltonian, as it must.)

For the remainder of this paper, we concentrate on a particular example, the harmonic oscillator with frequency

$$
\omega(t)=\frac{r}{t+s},
$$

where $r$ and $s$ are constants, with $|r|>\frac{1}{2}$. (We will see the importance of this restriction later.) This choice of $\omega(t)$ ensures that the Hamiltonian

$$
\mathbf{H}=-\frac{\hbar^{2}}{2 m} \frac{\partial^{2}}{\partial x^{2}}+\frac{1}{2} m \omega^{2}(t) x^{2}
$$

satisfies the criterion (5) which we derived above. (Note that $s$ reflects the choice for the origin of time, and so, with a suitable choice, this is a system with frequency inversely proportional to time.)

With the change of variables

$$
y=\left(\frac{m}{\hbar(t+s)}\right)^{1 / 2} x
$$

(here we are absorbing extra factors of $\hbar$ and $m$ into $y$, as compared to (丰)), separation of variables with $y$ and $t$ will work. We find that

$$
T_{k}(t)=\left(1+\frac{t}{s}\right)^{-\left(i \gamma_{k} / 2\right)}
$$


The analogue of the stationary states for this system satisfy the ordinary differential equation

$$
-\frac{d^{2}}{d y^{2}} Y_{k}+i \frac{d}{d y} Y_{k}+r^{2} y^{2} Y_{k}=\gamma_{k} Y_{k}
$$

This equation is solved most easily by operator methods. The pseudo-Hamiltonian operator $\widetilde{\mathcal{H}}$ for this system is

$$
\widetilde{\mathcal{H}}=-\frac{d^{2}}{d y^{2}}+i y \frac{d}{d y}+r^{2} y^{2}
$$

Now define the ladder operators

$$
a=\frac{d}{d y}+\alpha y, \quad a^{\dagger}=-\frac{d}{d y}+\alpha^{*} y
$$

where $\alpha$ is a complex number. Choosing

$$
\alpha=r e^{-i \theta}, \quad \sin \theta=\frac{1}{2 r},
$$

(recall that $|r|>\frac{1}{2}$ ), we have the algebra

$$
\begin{aligned}
{\left[a, a^{\dagger}\right] } & =2 r \cos \theta \\
\frac{1}{2}\left\{a, a^{\dagger}\right\} & =\widetilde{\mathcal{H}}+\frac{i}{2} \\
{[\widetilde{\mathcal{H}}, a] } & =-2 r \cos \theta a \\
{\left[\widetilde{\mathcal{H}}, a^{\dagger}\right] } & =2 r \cos \theta a^{\dagger} .
\end{aligned}
$$

This algebra is clearly a generalization of the familiar algebra of the raising and lowering operators of the time independent harmonic oscillator.

From (19b), we see that $\gamma_{k}=\operatorname{Re}\left(\gamma_{k}\right)-\frac{i}{2}$, and that $\operatorname{Re}\left(\gamma_{k}\right) \geq 0$. Without loss of generality, we consider the case the $r \cos \theta>0$. In this case, we see that $a$ lowers and $a^{\dagger}$ raises the real part of the $\widetilde{\mathcal{H}}$ eigenvalues by $2 r \cos \theta$. Thus we see that there must be a "ground state" $Y_{0}$ such that

$$
a Y_{0}=0
$$

This yields that

$$
Y_{0}(y)=\exp \left(-\frac{1}{2} \alpha y^{2}\right),
$$

while the other states of this system are given (up to normalization) by

$$
Y_{k}(y)=\left(a^{\dagger}\right)^{k} Y_{0}(y), \quad k=1,2,3, \ldots
$$


Note that all our results reduce to the known results for the time-independent harmonic oscillator in the appropriate limit $(r \rightarrow \infty$, with $s / r$ finite).

We can evaluate the $\gamma_{k}$ from (15) using these raising and lowering operators. One readily finds that

$$
\begin{aligned}
\gamma_{k} & =\alpha+k\left(\alpha^{*}+\alpha\right) \\
& =-\frac{i}{2}+\left(k+\frac{1}{2}\right)\left(4 r^{2}-1\right)^{1 / 2}, \quad k=0,1,2, \ldots .
\end{aligned}
$$

Thus we have solved completely and exactly the quantum mechanical harmonic oscillator with frequency inversely proportional to time. All physically observable quantities may now be computed explicitly for this system.

In closing, we remark that we have identified time-dependent quantum systems for which separation of variables is a useful and effective technique. We have presented this work in one spatial dimension, but clearly the same methods can be applied in higher dimensions as well. In the case of the time-dependent harmonic oscillator, we have identified a new exactly soluble model. There are several areas appropriate for further development, including the application of these methods to field theoretic examples; the identification of solitons whose excitation spectrum can be found using the techniques we have described; and the interpretation of our results in a fundamental way in $x$-space. It would be particularly interesting to classify the exactly soluble models with the type of time dependence we have explored; indeed we anticipate that many familiar exactly soluble time-independent models have exactly soluble time-dependent generalizations.

\section{Acknowledgments}

J.R. acknowledges the support of a Hobart and William Smith Summer Student Research Grant. 


\section{References}

[1] E. Merzbacher, Quantum Mechanics, $2^{\text {nd }}$ ed. (John Wiley \& Sons, New York, 1970).

[2] P.A.M. Dirac, Nature 139 (1937) 323;

P.A.M. Dirac, Proc. Roy. Soc. A165 (1938) 199.

[3] N.H. Christ and T.D Lee, Phys. Rev. D12 (1975) 1606. 